\title{
Communicating Healthcare Provider Perspectives about Mi'kmaq Women's Access to Pap Smear Screening in Eastern Canada: A Qualitative Study
}

\author{
Catherine D. MacDonald ${ }^{1, *}$, Audrey Steenbeek ${ }^{2}$ \\ ${ }^{1}$ Rankin School of Nursing, Saint Francis Xavier University, Antigonish, Nova Scotia, Canada \\ ${ }^{2}$ School of Nursing, Dalhousie University, Halifax, Nova Scotia, Canada
}

Received March 22, 2021; Revised June 4, 2021; Accepted June 20, 2021

\section{Cite This Paper in the following Citation Styles}

(a): [1] Catherine D. MacDonald, Audrey Steenbeek, "Communicating Healthcare Provider Perspectives about Mi'kmaq Women's Access to Pap Smear Screening in Eastern Canada: A Qualitative Study," Universal Journal of Public Health, Vol. 9, No. 3, pp. 149 - 161, 2021. DOI: 10.13189/ujph.2021.090306.

(b): Catherine D. MacDonald, Audrey Steenbeek (2021). Communicating Healthcare Provider Perspectives about Mi'kmaq Women's Access to Pap Smear Screening in Eastern Canada: A Qualitative Study. Universal Journal of Public Health, 9(3), 149 - 161. DOI: 10.13189/ujph.2021.090306.

Copyright $\bigcirc 2021$ by authors, all rights reserved. Authors agree that this article remains permanently open access under the terms of the Creative Commons Attribution License 4.0 International License

\begin{abstract}
Indigenous women have higher rates of cervical cancer and lower rates of Papanicolaou (Pap) smear screening than non-Indigenous in Canada. Healthcare providers are well positioned to address the inequities in access to Pap screening that many Indigenous women continue to experience. The purpose of this study was to explore healthcare providers' experiences and perspectives about Mi'kmaq women's access to Pap smear screening in Eastern Canada, and to consider the broader historical, economic, and sociopolitical contexts that shape healthcare experiences. The methodology is a qualitative, community-based participatory action research approach that embodied Indigenous approaches to knowledge development and post-colonial theoretical perspectives in a two-eyed seeing approach. In-depth semi-structured in-person interviews were conducted with five healthcare providers twice, in two First Nations communities in Nova Scotia. Findings revealed two major overarching themes: a) Understanding the Realities of Indigenous Women's Lives; which involved recognition of the impact of history, and socioeconomic and political factors; and b) Fostering Indigenous Women's Access to Pap Smear Screening, through fostering trusting relationships, cultural safety, addressing confidentiality issues and optimizing processes to improve experiences. Conclusions indicated approaches to foster Mi'kmaq women's access to Pap screening included a social
\end{abstract}

women's day, targeting youth, explaining step-by-step during screening, providing Pap results to women and having nurse practitioners perform screening. Implications for practice comprised addressing confidentiality issues in community health centres, involving the community in Pap screening decision-making, acknowledging the impact of historical trauma on access to screening and optimizing processes to address and improve inequitable access to screening. This study highlights the importance of healthcare providers' participation in understanding and encouraging Indigenous women's access to Pap smear screening. Healthcare providers need to consider a wider view of the realities of Canadian Indigenous women's lives, and acknowledge the impact of historical trauma, socioeconomic factors, and racism on women's accessing of Pap smear screening. Healthcare providers can implement collaborative and culturally safe screening approaches, while acknowledging Indigenous community strengths to improve inequities in healthcare access and health outcomes.

Keywords Healthcare Providers, Pap Smear Screening, Mi'kmaq Women, Community-Based Participatory Action Research, Two Eyed-Seeing Approach, Qualitative Research 


\section{Introduction}

Healthcare providers are often the first point of entry to the healthcare system and advocates "for best practices in developing strong Aboriginal ${ }^{1}$ communities" [8]. Their approaches and interactions with Indigenous people can influence current and future accessing of healthcare services [42], such as Pap smear screening. Canadian Indigenous women continue to experience higher rates of cervical cancer and have lower rates of Pap smear screening than non-Indigenous women $[2,18,65]$. It is reported that Indigenous women are 2 to 20 times at risk for being diagnosed with cervical cancer in Canada [33] and mortality rates are approximately 2.5 to 10 times higher among this population [10, 20, 29]. Similarly, compared to non-Indigenous women, American Indian and Alaska Native women have a higher incidence of cervical cancer, are diagnosed at a later stage and have nearly twice the mortality rate from cervical cancer [56].

The multiple jurisdictions involved in Canadian Indigenous health funding makes healthcare services complex and funding challenging in First Nation communities [24]. Federal, provincial, and local jurisdictions are responsible for multiple portfolios related to Indigenous people's health, including Pap smear screening. The extent to which Pap screening is prioritized in comparison to other health programming initiatives such as diabetes, HIV/AIDS, healthy child development, and immunizations is concerning [23].

Healthcare providers are well positioned to address Indigenous women's inequities to accessing Pap smear screening services, by acknowledging the social determinants of health and the impact on of Indigenous women's lives. It is also vital to recognize the impact of historical trauma and violence experienced by Indigenous women, while being aware that they are resilient and have the strengths and capacities to survive violence and trauma $[11,34,66]$. It is well known that historical trauma was experienced by the Indigenous peoples of Canada, the United States, Australia and New Zealand [6, 25, 27]. Historical trauma is a cumulative process of emotional and psychological wounding and suffering, that occurs over the lifespan and across generations, that emanates from massive group trauma [12]. Historical trauma has been directly related to colonization, residential schooling and assimilation; resulting in cultural and identity loss [58, 62]. Domestic violence, emotional trauma, child abuse, alcoholism and drug abuse are well documented effects of historical trauma [12,37].

This paper reports findings from a qualitative community-based research study exploring Mi'kmaq ${ }^{1}$

\footnotetext{
${ }^{1}$ The Mi'kmaq are the founding people of Nova Scotia and remain the predominant Aboriginal group within the province. The Mi'kmaw nation has existed in what is now Nova Scotia for thousands of years, and is made up of thirteen Bands/First Nations, each of which is governed by a Chief and Council (Office of Aboriginal Affairs, 2011).
}

women's experiences with Pap smear screening in the province of Nova Scotia in Eastern Canada. The analysis of stories related to Pap smear screening from healthcare providers working with Mi'kmaq women in two First Nation's communities will be presented. Two major themes that emerged from their stories are: a) Understanding the Realities of Indigenous Women's Lives and b) Fostering Indigenous Women's Access to Pap Smear Screening. Findings from the same study that focused on the Mi'kmaq women participants were previously published [36].

\section{Methods}

\subsection{Design}

This qualitative research inquiry used participatory action research (PAR) that was informed by postcolonial feminist theoretical perspectives and drew on Indigenous principles in a "two-eyed seeing" approach [26]. The use of two-eyed seeing means that traditional knowledge (as an Indigenous approach to knowledge development) and western science are considered complementary knowledge forms, that when used in combination assist in responding to questions about the health of Indigenous people and communities [26, 38]. Also, the Indigenous principles of relationality, respect, reciprocity, inclusivity, relevance, and responsibility were adhered to and helped to shape the study $[9,31,59,61]$. This research design provided a means for analyzing and interpreting how the colonial past and the current socioeconomic and political climate impacts Indigenous women's access to Pap smear screening, and further understanding of healthcare providers' perceptions as to the reasons why some of these women may not be accessing Pap screening services.

Postcolonial theoretical perspectives provided a lens for critically analyzing the interwoven contextual complexities that influence Indigenous women's health and the factors that shape inequities to accessing Pap smear screening services $[3,15,47]$. Postcolonial feminism also addressed issues of gender, differences in ethnicity, and life circumstances, which impacted Indigenous women's access to healthcare services [30, 60]. PAR principles of equity, social justice, democratic, collective decision-making and reciprocity $[31,35,55]$ were used throughout this research. PAR principles were maintained by enabling the participation of community members and healthcare providers, valuing all participants' viewpoints, using collaborative decision-making throughout the research process, developing relationships and fostering open communication. Consent forms were signed by the participants. Ethical approval to conduct the study was obtained in writing from Dalhousie University Health Sciences Human Research Ethics Committee, 
Mi'kmaq Ethics Watch Cape Breton University, and Mi'kmaq community leaders. The Canadian Institute of Health Research (2007) guidelines for health research involving Aboriginal people and the OCAP (ownership, control, access, and procession) principles (2004) were also employed.

\subsection{Community Settings}

The settings for this study included two small rural Mi'kmaq First Nations communities in the province of Nova Scotia on the east coast of Canada, with populations that ranged in size from $540-943$ people. These communities had similar characteristics to other Indigenous communities in Nova Scotia with respect to language and access to healthcare services. Some Indigenous communities in Nova Scotia have kept their traditional language, while some communities have not. One of the First Nation communities in this study has kept the Mi'kmaq language alive by teaching the children at the daycare words and phrases in Mi'kmaq. In the second First Nation community, there were few individuals that spoke Mi'kmaq fluently.

\subsection{Data Collection}

\section{Recruitment of Healthcare Providers}

Healthcare providers were recruited using purposeful sampling. Community facilitators in each community assisted with recruitment of participants, acted as a community liaison between the community and the researcher, provided feedback regarding the interview guide and research process, and reviewed and commented on themes throughout the analysis. The community facilitators in each community provided names of potential healthcare provider participants. The researcher then contacted potential healthcare participants in case the community facilitators were not comfortable with contacting them and to balance power differentials that may have existed. The total number of potential healthcare providers that could have been interviewed in both communities participated in the study. Four healthcare providers were contacted in person while visiting the healthcare centers and were given an information letter explaining the study and their interest to participate in the study determined. Another healthcare provider was invited to participate by telephone. All five agreed to participate in the study, as they believed it was a topic that was important in Mi'kmaq communities.

The healthcare providers included physicians, registered nurses, and a nurse practitioner, none of whom identified as Indigenous or lived in of the communities involved in this study. Healthcare providers had between three to more than 20 years' experience working with Indigenous people. None of the healthcare providers worked in both Mi'kmaq communities. Informed consent was obtained after the study was fully explained.

\section{In-depth Interviews}

There were nine audiotaped semi-structured in-depth interviews conducted by the first author. Healthcare providers could choose to participate in one or two 60-90 minute in-depth interviews about their experiences with providing healthcare, particularly Pap smear screening services, to Indigenous women. Healthcare providers were asked for their perspectives on the reasons why Indigenous women may or may not be accessing screening services, their recommendations for how to achieve equitable access to screening services, and the nature of the knowledge and or expertise required for providing Pap screening services. Each healthcare provider completed a brief demographic form that asked about the number of years they had worked in the community, their experience working with Indigenous people, employment status, and healthcare provider role.

A second interview took place after each initial interview was transcribed with all but one of the healthcare providers, for the purpose of reviewing transcripts and expanding and providing clarity on what they said in the first interview. After each interview field notes detailing thoughts, observations and reflections about what was said, how it was said, verbal and non-verbal communication, and the environment were recorded. Some of the field notes assisted with the planning for follow-up questions in the second interviews and proved helpful when interpreting data.

\subsection{Data Analysis}

Transcripts were transcribed verbatim, compared with audio-recordings for accuracy and imported into N-Vivo qualitative software (QSR International, 2007). Thematic analysis processes described by Sandelowski [48] and O'Connor and Gibson [43] were employed to identify themes from the participants' stories. Healthcare providers provided input into the themes and sub-themes and offered some feedback regarding the theme titles that emerged from the data. The primary researcher coded the data and two co-supervisors reviewed some of the coding processes to ensure validity of themes and subthemes. Trustworthiness of the study was enhanced by member checking, recording field notes, and by forming partnerships with and engaging community facilitators in the research process. (Table 1: A description of the thematic analysis process)

\section{Results}

Two major themes were identified from healthcare providers' stories: "Understanding the Realities of Indigenous Women's Lives" and "Fostering Indigenous Women's Access to Pap Smear Screening." Each theme and its sub-themes (Table 2) are discussed along with 
supporting quotes.

\section{"Understanding the Realities of Aboriginal Women's Lives"}

To understand the complex reasons why some Indigenous women may not be accessing Pap smear screening services, healthcare providers communicated the importance of considering a wider view of the realities of these women's lives. Within this theme, two sub-themes emerged wherein healthcare providers talked about the importance of acknowledging the impact of historical trauma, socioeconomic factors, and racism on women's accessing of Pap smear screening. The two sub-themes are: a) History and b) Socioeconomic and Political Factors.

\section{History}

This sub-theme describes the healthcare providers' stories about the importance of understanding the impact of history on Indigenous women's lives and its impact on their accessing Pap smear screening services. All of the healthcare providers talked about historical trauma, the impact of residential school and colonization with Indigenous people. One healthcare provider described "the lives and health of Aboriginal people prior to colonization as being much better than it is now and that it was important for healthcare providers to consider the impact of colonization on the lives and health of Aboriginal people, rather than blaming them for their ill health, lifestyle choices, and for not accessing Pap smear screening services." Another healthcare provider described, "the violation of treaties and the colonization of Canadian Aboriginal people. Being historically disempowered and experiencing the deceptions associated with the violation of signed treaties has negatively impacted many Indigenous people's trust in western systems, particularly healthcare systems and their sense of well-being." Although every healthcare provider communicated the importance of understanding residential schools' impacts on Indigenous peoples' health and access to healthcare, many of their narratives tended to regard all Indigenous people as suffering in the same ways as a result of residential schools. A few other healthcare providers directly associated the historical trauma of residential schools with the lack of self-care and the lack of accessing Pap smear screening. A healthcare provider stated, "Not caring for oneself. Not valuing oneself due to historical trauma."

Even though healthcare providers communicated the importance of acknowledging the impact of historical trauma on Indigenous women's lives and access to Pap smear screening services; the healthcare providers tended to have varying degrees of knowledge and understanding. Healthcare providers with more experience working in
Indigenous communities seemed to have a more in-depth understanding of the diversity in experiences and impact associated with historical trauma on the accessing of the healthcare, including screening services.

\section{Socioeconomic and Political Factors}

Participants talked about women living in poverty, lacking childcare and transportation, lacking access to education, and having multiple roles and responsibilities. They communicated that even when these women did access healthcare services some experienced racism and stereotyping, which negatively impacted future access.

Almost all of the healthcare providers communicated that the loss of parenting skills, mental health issues, suicides, addictions, and abuse that they observed in the communities was an outcome of generations of historical trauma resulting from the impacts of residential schools and colonization. The fracturing of the family and loss of parenting skills were highlighted by healthcare providers as being extremely influential in affecting Indigenous women's access to Pap smear screening and other healthcare services. Historically, the family was central in health education and teaching young girls about their bodies and caring for their bodies by passing knowledge from one generation to the next by way of storytelling. As a result, of residential schools and colonization, teaching and information about the body had been lost [7]. One shared, "When people were in residential schools, information got lost about all those life skills".

Healthcare providers talked about historical trauma with regards to the violation of land treaties; losses of language, traditional medicines, foods, and parenting skills; mental health issues; addictions and abuse; and lack of self-care, all of which are supported by literature [21-22, $28,46]$. Stories of resiliency and strengths of these women were rarely mentioned in this study. Arnold and Bruce [5] have noted, "It is difficult to find information on the inherent strengths and abilities of First Nations' populations for their own health and healing" (p. 260). It is also important to be aware that not all Indigenous people experienced or continue to experience historical trauma in the same way and although many people were confronted with historical trauma, there are Indigenous people that have maintained their identities, languages, cultures, and traditions [39].

\section{Living in Poverty}

Living in poverty was cited by healthcare providers as being one of the major reasons for Indigenous women not accessing Pap smear screening services. When asked what was perceived to be the major issues for women accessing Pap smear screening services one healthcare provider responded by saying, "It would be economic, it would be historical, poverty driven definitely for a lot of them." 
While this participant noted that poverty was a major issue preventing Indigenous women from accessing Pap smear screening, she/he had a broader perspective, linking poverty with history, and did not blame Indigenous women for being poor or living in poverty.

Three of the healthcare providers also pointed out that a major issue confronting poor or low-income Indigenous women was the lack of affordable and accessible childcare services for women seeking services outside the community. Another participant related that being poor was a variable that increased women being marginalization by mainstream society and the medical community. A few others talked about the high rates of teen pregnancies in the communities. One provided details about the economic support and security offered by the community to care for many of the pregnant youth.

\section{Transportation}

Healthcare providers identified that Indigenous women living in poverty experience issues with transportation and childcare, which often impacts their access to health services. One explained, "There are transportation issues. We have a lot of issues here with medical transportation and transportation to other healthcare facilities." Not only are there problems with lack of available transportation, another fundamental issue revealed was that even when transportation exists in the community, the driver, referred to locally as "the carrier," may be related to you and want to know why you are going to a certain appointment.

Another reported barrier was that drivers, or carriers, are required to complete medical forms that state the reason for medical transportation. A healthcare provider explained to be paid for driving to the appointment, the medical drivers require a form to be filled out by the healthcare provider and although the information provided is vague, it is still information that the patient potentially might not want the driver to know. In many instances, the driver knowing the women will inquire in casual conversation as to why they are attending a healthcare clinic or physician's appointment. Healthcare providers indicated that many women felt obligated to tell why they were going for healthcare when questioned because not responding may be viewed as being disrespectful.

\section{Education}

A few healthcare providers discussed how education is valued in Indigenous communities, but there was a lack of access to education because of poverty. One healthcare provider talked about education as being primarily designed for "white women." While this participant noted that Indigenous people do not have the same access to education as "white middle class" people, healthcare providers still expect to educate Indigenous people using "white" ways and tools, which does not seem reasonable or appear to work.

While there was some recognition that awareness of the importance of Pap screening was improving, most healthcare providers indicated that Indigenous women lacked knowledge and education about Pap smear screening. Healthcare providers pointed out that it was part of their responsibility to educate women and heighten awareness in the community about Pap smear screening to increase access. A few of the healthcare providers emphasized that being poor and lacking education has led some women to not value their health and accounts for women not accessing Pap smear screening.

All of the healthcare providers shared a genuine interest in working with Indigenous women and told stories about the impact that socioeconomic factors had on their access to healthcare services, including Pap smear screening. Poverty influenced not only access to health, but also access to education, which in turn impacted access to health and health outcomes.

\section{Racism and Stereotyping}

The participants frequently mentioned racism and stereotyping. For example, "Some First Nations people may say that they've felt discriminated against or had experience with racism when they went to the health care system, and that's why that prevents them from follow-up care and what not."

Healthcare providers discussed their perceptions of Indigenous people. One communicated that she had some preconceived ideas before coming to the community to provide Pap smear screening and other healthcare. "I think I had some preconceived ideas before I started working in that particular community and I didn't really necessarily know exactly what the needs were going to be," she/he stated.

Healthcare professionals talked about the racism, discrimination, "Othering" 2 and essentializing that Indigenous people experience when accessing healthcare services, which is supported in literature $[3,10,56]$. Their narratives revealed their perceptions of Indigenous people are expressed in words and/or actions that facilitate or further marginalize them from accessing mainstream health services. An example of this type of labeling and essentializing, which often goes unrecognized, can be seen in the following quote from one of the healthcare providers: "I just think the substance use in that particular culture and that particular community seems to start really young ... It's part of their culture."

Healthcare providers' stories contained indications of their attempts to counter cultural assumptions and white middle-class values by trying to understand the broader

\footnotetext{
2 Othering is referred to as "the projection of assumed cultural characteristics, "differences", or identities onto members of particular groups" (Browne, Smye \& Varcoe, 2005, p. 21)
} 
historical, socioeconomic, and political contexts that affected women's lives. Most of the healthcare providers acknowledged that negative and discriminatory assumptions and stereotypes about Indigenous people exist and that they originate from societal middle-class values, biomedical perspectives, and the legacy of colonialism.

\section{Fostering Aboriginal Women's Access to Pap Smear Screening}

The theme, "Fostering Aboriginal Women's Access to Pap Smear Screening" with four sub-themes, described what healthcare providers in this study considered important and would thereby endorse and recommend as a means of increasing Pap screening. These recommendations are based upon healthcare providers' experiences and knowledge acquired while working in Indigenous communities. The sub-themes include: a) Trusting Relationships; b) Cultural Safety in Practice; c). Confidentiality Issues; and d) Optimizing Processes to Improve Experiences

\section{Trusting Relationships}

Participants described the value and importance of building respectful relationships with Indigenous people based on trust and the positive impact this has on healthcare access. For many historical and contemporary reasons, building trusting relationships takes time and patience. One participant described the importance of developing a trusting relationship before initiating healthcare, such as Pap smear screening. She/he communicated that respect, connection, relationship, and family are very important values held in high esteem in Indigenous communities. Having a full-time nurse working in the community provided an opportunity to develop trusting relationships, which increased women's access to Pap smear screening. Another described building trusting relationships as a strategy to recruit other women in the community to access healthcare services.

\section{Cultural Safety in Practice}

All of the healthcare providers perceived that their education or knowledge about cultural safety dramatically affected Indigenous women's access to Pap smear screening. Healthcare providers' stories comprised accounts of their learning from the community by experience or by attending community ceremonies and events. Healthcare providers indicated they had learned about culturally safe and appropriate practices from community members, not in their formal education, and described "the community as their teacher." Healthcare providers also acknowledged that community members such as Elders were their primary teachers about
Aboriginal history, traditions, and knowledge.

All of the healthcare providers either explained or alluded to the importance of having cultural safety, cultural competence, or cultural sensitivity in their formal education, so that they would be competent to provide healthcare. Some made the point that you cannot learn cultural safety in its entirety in formal education, and that some of this learning occurs in informal and formal ways in the community. Healthcare providers can learn about cultural safety informally by encouraging the people they work with "to be open to saying and telling you about what is culturally appropriate or not."

\section{Confidentiality Issues}

Every healthcare provider communicated the importance of maintaining confidentiality and reported that part of their role as a healthcare provider was to ensure that confidentiality was maintained. Notably, almost all of them identified challenges with maintaining patient confidentiality in community health centers due to lack of proper procedures and policies for housing patient data. In addition, the way personal information is housed has led to breeches in confidentiality and some community members employed at the health centre have access to personal information about other community members.

Although healthcare providers identified that maintaining confidentiality is part of their role and code of ethics, one articulated, "sometimes healthcare providers or staff in the health center breeched confidentiality unintentionally in casual conversations or social settings." For example, they might mention seeing an individual at the health center. Because the community is small and close-knit, information breeches such as this can create "a real barrier to some of the access of care for Aboriginal community members."

A few healthcare providers pointed out the perspectives regarding confidentiality within Indigenous communities differ from mainstream perspectives. For example, "in certain situations that involve the community, healthcare providers are expected to discuss healthcare issues with Elders and/or family members, which contrasts mainstream healthcare policies and protocols."

\section{Optimizing Processes to Improve Experiences}

This sub-theme describes what the healthcare providers in this study recommend to improve Indigenous women's experiences with and access to Pap smear screening. All of the healthcare providers in the study shared practices to improve Pap smear screening experiences and access to services.

Community input. Healthcare providers suggested that community members, especially women, should have input into Pap smear screening education and 
recommendations to improve screening and access. In addition, they indicated women should have a choice about who will perform their Pap smear screening. Consulting with communities is not only respectful, but enables healthcare providers to develop trusting relationships with community members, which in turn assists in gaining knowledge and fostering solutions to accessing Pap smear screening services.

Although one talked about the importance of women having input and choice into Pap smear screening education, it was recommended that having workshops in the community was one way to increase access to screening. Yet, she cautioned that the women must be offered choice in what they want to be taught in the workshop by healthcare providers. She also communicated that until recently, the community did not have much input into what they wanted in regards to Pap smear screening and other healthcare services.

Some healthcare providers identified that the gender of the healthcare provider offering Pap smear screening was important to consider, particularly for women with a history of sexual abuse. One healthcare provider stated “maybe they have a history of abuse or maybe they're just uncomfortable having a male do their Pap in general, just because it's a very personal thing. They don't want that relationship with a male provider. They want a female provider doing that procedure with sensitivity."

However, other healthcare providers suggested that for Indigenous women who have been sexually abused, the issue is not the gender of the healthcare provider doing the Pap smear screening that is important. Rather it is having continuity with the same healthcare provider performing the Pap screening over time. For example, "I think by the nature of how invasive it is into their private space, if they've been abused they may not disclose it up front. And I think that's the biggest issue for them is not knowing and not always having the same person ... It does not need to be a female doing the Pap."

More convenient and accessible Pap screening. Some of the healthcare providers told stories that included references to Indigenous women needing more convenient and accessible Pap screening services in the community. This was commonly perceived by healthcare providers as a strategy to improve overall Pap smear screening rates. According to healthcare providers, by offering more convenient and accessible services in the communities, barriers such as the lack of childcare and transportation would be reduced, thus making access more possible for women. One healthcare provider stated:

"So, they need to have these services in their community and they need to build an infrastructure that involves the women of the community to develop a program that is responsive to their needs and their own policies."

A few other healthcare providers also recommended that the timing of appointments impacts women's access to screening. One suggested that healthcare providers need to offer more convenient after-hour clinics and clinics more regularly so that women who are working or in school can attend screening. Some participants identified that increasing screening services in the community was the solution to increase the rates of access. However, other healthcare providers recognized that the complexities of the women's lives were such that improving access to Pap screening required multiple strategies.

\section{Approaches to Improve Service Delivery}

Healthcare providers suggested various innovative approaches to improve service delivery for Indigenous women and empower their access of Pap screening

"A Social Women's Health Day." Some healthcare providers in both communities described that making Pap smear screening a social event with food was a successful innovative approach that they had implemented. They reported improved accessing of screening services and increased awareness and knowledge about Pap smear screening. It was suggested:

Having an open house for First Nations women so they could come in one afternoon and have tea and see [healthcare providers] and increase awareness about Pap screening socially.

One recommended having “A Social Women's Health Day" that included food and prizes to empower women to have screening completed. During this event, women were provided ample opportunity to voice what they wanted to know about Pap smear screening and to talk about issues they had with access or experiences. Women were able to make an appointment for future screening or have screening done that day if possible. Women could bring their children if they desired; thereby eliminating the need for childcare. One healthcare provider added, "that having social Pap clinics also benefits healthcare providers' too because it increases their knowledge of the traditions and challenges within the community."

Targeting youth. It was suggested by some healthcare providers, that having innovative Pap smear screening educational sessions, such as "Get a Pap, Win an iPod" that target young girls in the community was an important strategy to increase screening. Having these types of sessions is a way not only to increase access, but also increases young women's knowledge and awareness about Pap smear screening. One healthcare provider reported that normalizing Pap smear screening for youth might set a trend for them to attend regular screening.

Other healthcare providers acknowledged that young Indigenous women, required education about the procedure, screening guidelines, reasons to access Pap smear screening, and results. They recommended targeting the youth in school in the hopes of increasing their decision to access screening and to get into a routine of regular screening. 
One perceived that having more Indigenous based educational and teaching tools at the health centre where young women often drop in may be a way to encourage women to attend screening. It was suggested that seeing a young Indigenous woman's face on a pamphlet or brochure may also inspire other Indigenous women to attend screening. Further, as women become educated about Pap smear screening they, in turn, could inspire and encourage other women to go for screening.

Going step-by-step and providing Pap results. Going step-by-step during Pap smear screening and informing women of results were other approaches identified by some healthcare providers to improve experiences and increase their attendance at regular screening. Healthcare providers revealed the importance of talking women through screening to decrease fears and anxiety, while building trust. Healthcare providers that did go step-by-step with women throughout the Pap screening procedure relayed that women appeared more comfortable and that knowing what to expect next alleviated women from tensing up, thus decreasing discomfort. Some talked about the importance of step-by-step coaching during the procedure itself, especially if women have been sexually abused in the past.

One explained that "I'm totally coaching the whole time I'm doing the procedure."

A few of the healthcare providers discussed the importance of informing Aboriginal women of their Pap smear screening results. One revealed that it is part of her role to inform and educate her patients of all test results, whether positive or negative, as well as to ensure they understand about follow-up care. Also, provided them with a timeframe of when to expect results to reduce anxieties during the wait. However, other healthcare providers talked about the impracticalities of calling all women with negative results and that this is not an expected part of the usual standard of care. One went on to explain that calling women with results has its own challenges.

NPs and Registered Nurses to perform Paps. Over half of the healthcare providers suggested that having a nurse practitioner or a registered nurse who works in the community and performs Pap screening would provide better access to and quality of screening services. Further, a nurse practitioner or registered nurse in the community would develop relationships in the community, know the health histories of the women, and have a vested interest in the health and well-being of the women. In addition, they would allocate time to educate women about screening and results in a manner in which women can understand. One stated, "I really see a role for either a family practice nurse or a nurse practitioner. I think they would provide a lot of expertise that physicians wouldn't provide in terms of community assets and I see that as perfect for access [to healthcare]." For other health care providers, the concern was getting Pap smear screening completed, not who did the screening.

Funding, resources, supplies, and services. Healthcare providers communicated that there exists a lack of available funding, resources, and supplies for education and Pap smear screening in Indigenous community health centers, which posed ongoing challenges for women wanting to access screening in their community. Providing adequate funding was highlighted as being essential for ensuring access to screening services. It was recommended that policy-makers and government develop innovative ways to reallocate or increase resources for Pap smear screening supplies, educate Indigenous women about screening, educate healthcare providers to perform screening, and increase screening services offered in communities. One commented, "There isn't money in that community for Pap smear screening. We take all our own equipment and they use their nurse [community health centre] on that day we're down there." For some healthcare providers, not being able to count on having supplies and equipment for Pap smear screening in the community meant that they did their screening in locations outside of the community.

Another talked about the lack of follow-up services for women that have been sexually abused. Although acknowledged by healthcare providers that sexual abuse and violence exists in the communities, the issue is not being addressed during screening. One commented, "healthcare providers often do not ask about sexual abuse during Pap screening due to time constraints and it is not a question on the standard recording form used in a healthcare visit." Also, it was indicated there are not adequate supports in place for healthcare providers to address or deal with sexual abuse and this was another reason they did not ask questions regarding abuse prior to or during Pap smear screening. Many healthcare providers recognized the impact of a history of sexual abuse on women's experience during Pap smear screening, and at the same time knew that time constraints and lack of adequate supports limited the capacity to address the ongoing psychosocial needs of women who have been sexually abused. Therefore, healthcare providers often do not ask women the question. This can be an ethical dilemma for healthcare providers. They know the importance of inquiring about abuse to enable healing and intervene in the cycle of abuse; yet know they do not have the resources to allocate to follow up with these women. Not being able to provide follow up to help women deal with the pain and suffering associated with abuse, leaves healthcare providers hesitating to ask the question.

\section{Discussion}

Healthcare providers' perceptions of Indigenous people can either facilitate women's access to healthcare services or further marginalize Indigenous women from accessing 
healthcare systems [40]. Healthcare providers in the study described the importance of building relationships with Indigenous women for understanding Pap smear screening from women's context. Relationship building is particularly important considering the marginalization, subjugation, and control Indigenous people experienced and the mistrust in relationships that ensued, and continues today because of colonial practices and government assimilation policies [52]. This in turn influences relationships between Indigenous and non-Indigenous people and further promotes social, economic, and political inequalities that affect the health and access to health for Indigenous peoples [52]. Healthcare professionals need to be cognizant of the power imbalances that oppress Indigenous people and communities, promote the valuing of differing worldviews, and employ a two-way process of teaching and learning that fosters new levels of understanding [1]. Healthcare providers in the study demonstrated a genuine interest in working with Indigenous women and identified the importance of understanding the impact that history, socioeconomics and politics play in shaping Indigenous women's lives and access to Pap smear screening. Healthcare providers working with Indigenous people must have at least a basic understanding of the disruptive impact that historical trauma has on the health and well-being of the specific Indigenous people with whom they are working [17, 22]. According to Young et al. [64] healthcare providers require knowledge and understanding about Indigenous communities and cultural beliefs, practices, and values. Thus, healthcare providers require education to develop a cultural consciousness to work with Indigenous people and implement cultural safety into their practice [1, 16]. Almost all of the healthcare professionals reported a lack of formal education regarding Indigenous people and their history, culture, economics, knowledge, medicines, healthcare, or healthcare access in the curricula of their professional programs. This finding is cohesive with the literature [1, $29,42,44,51]$. The healthcare providers also identified the community as their teachers for educating them about Indigenous people and health. Seeking education and knowledge from the community, particularly Elders, is also supported in the literature. Weber-Pillwax [59] reinforced that Elders are respected teachers and historians who guide individual and collective development.

Healthcare providers need to consider the social determinants of health and the broader contexts of Indigenous women's lives when considering why they may or may not be accessing Pap smear screening. Fostering Indigenous women's access requires the building of trusting relationships, cultural safety in practice, and the addressing of confidentiality issues. Every participant communicated the importance of building a trusting relationship to foster Indigenous women's access to healthcare services and Pap smear screening, which is also supported in the current literature $[10,14,41,42,50,52,63]$.

Black [10] suggests that healthcare providers organize and present health conferences and workshops in the Indigenous communities, take time to visit the communities, and hold Pap smear screening events that are focused on Indigenous women's health using Indigenous teaching tools with role models. This is congruent with findings from this study. Building women's capacity by increasing their knowledge about Pap smear screening may potentially increase their access to Pap smear screening services [41, 52].

Confidentiality in Indigenous communities was associated with culture by a few healthcare providers. Lack of confidentiality was attributed to not having proper procedures and policies for housing personal information and data, as well as the employment of community members at the health centres.

The extent of the discrimination and stereotyping experienced by Indigenous people is well documented in the literature $[13,16,54]$. The healthcare providers acknowledged that racism and stereotyping in mainstream healthcare and healthcare encounters exists, and that discrimination originates from societal middle-class values and biomedical perspectives. Yet, few talked about their own positions of privilege and power when talking about Pap smear screening encounters with Indigenous women. Most healthcare providers in this study continued to indicate that Indigenous women lacked knowledge and education about Pap smear screening with little recognition about the lack of available Indigenous tools and information to educate women.

Healthcare providers identified a number of approaches to foster Indigenous women's access to Pap screening such as having a social women's day, targeting youth, going step-by-step during screening, providing Pap results to women and having nurse practitioners to performs Pap screening. The lack of available funding, resources, and supplies for education and Pap smear screening in Indigenous community health centers will need to be addressed, if these recommendations were to be enacted. Similarly, strategies are needed to address confidentiality issues relating to housing of personal data in community health centres and confidentiality concerns associated with the current method of providing transportation to healthcare services outside the community. By fostering collaborative relationships with Indigenous women and communities, offering more convenient and accessible Pap smear screening services, providing culturally safe education and practices and involving the community in decision-making regarding Pap screening will improve screening rates. It is imperative that healthcare providers understand the realities of Indigenous women's lives by acknowledging the impact of historical trauma, socioeconomic factors, and racism on women's accessing 
of Pap smear screening services to implement collaborative and culturally safe screening approaches. Based on the study findings and the current literature, the authors include some recommendations for Pap smear screening practices and education required by healthcare providers to assist in performing Pap smear screening with Indigenous women. (Refer to Table $2 \& 3$ ).

\section{Conclusions}

This qualitative community-based participatory action research study explored healthcare providers' experiences and perspectives about Mi'kmaq women's access to Pap smear screening in Eastern Canada. Healthcare providers communicated the importance of taking a wider view of the historical context and the realities of Indigenous women's lives, to understand their reasons for accessing or not accessing Pap screening services. Healthcare providers also acknowledged the impact of socioeconomic factors on women's health and well-being, and the impact of colonialism on the loss of culture, language, traditional knowledge, and the fracturing of family units as stemming from historical trauma. However, it was less common for healthcare providers to focus on the positive aspects of community life, or how women exhibited resistance and resiliency, and exerted their agency in spite of experiencing historical trauma and multiple losses. It is essential for healthcare providers not only to gain understanding of the multifaceted impact of historical trauma as it pertains to Indigenous health, but also to understand and encourage women's and community strengths to improve inequities in healthcare access and health status. Healthcare providers must also acknowledge their lack of knowledge related to Indigenous people, yet acknowledge Indigenous women's strengths and knowledge related to Pap smear screening.

Table 1. Healthcare Provider Themes and Sub-themes

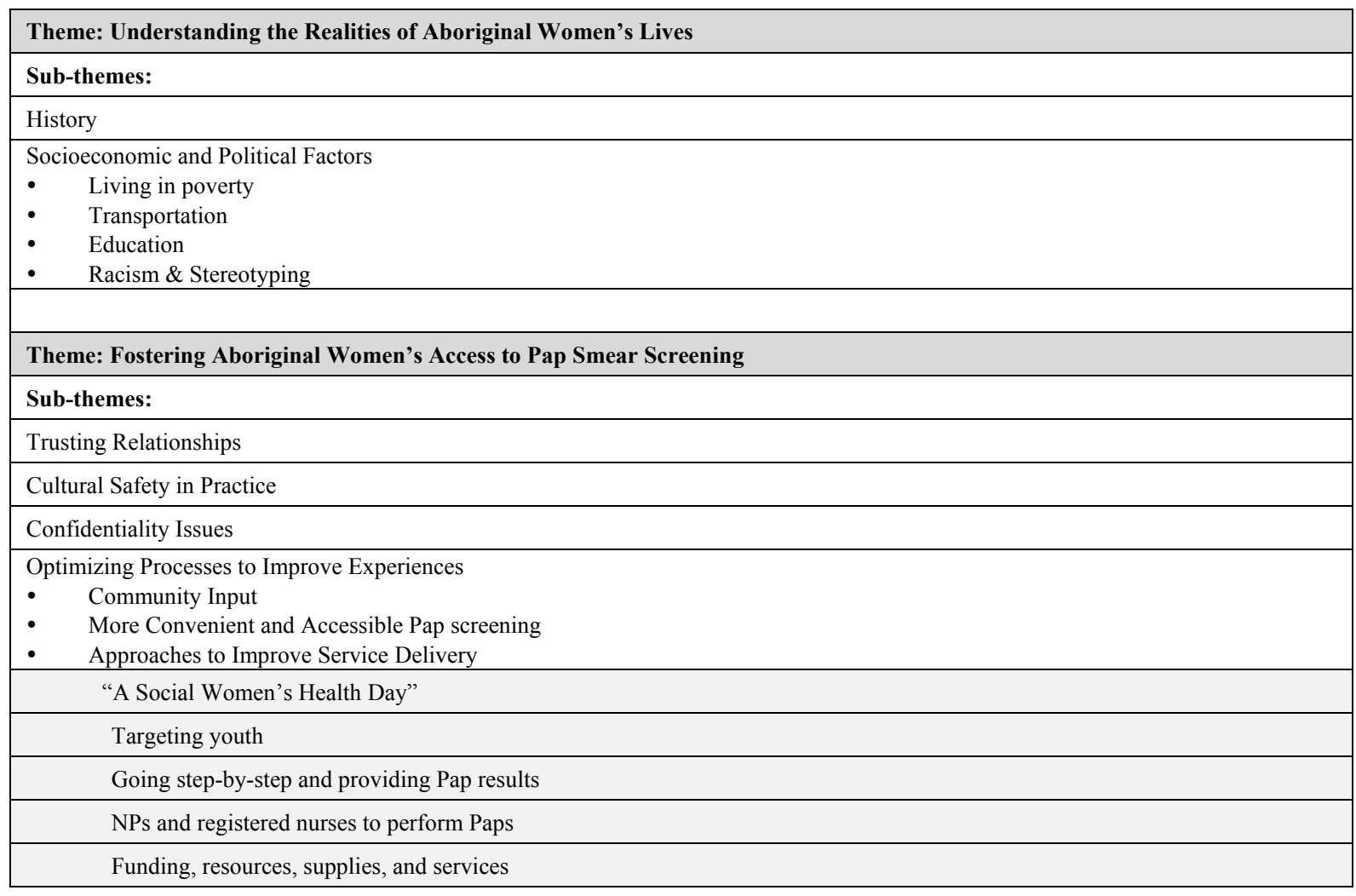


Table 2. Recommendations: Pap smear Screening Practices with Indigenous Women

\begin{tabular}{|l|}
\hline become familiar with and implement the concept of cultural safety into Pap smear screening practice \\
\hline develop respectful relationships with women and communities prior to initiating screening. \\
\hline $\begin{array}{l}\text { use relational and collaborative approaches to Pap smear screening by creating safe spaces for open dialogue to clarify questions and share } \\
\text { information about Pap smear screening and HPV prevention }\end{array}$ \\
\hline $\begin{array}{l}\text { learn about the principles of trauma-informed care, which can guide approaches to interacting with women in the context of intimate health } \\
\text { interventions, such as Pap smear screening }\end{array}$ \\
\hline provide Pap smear screening education that is framed in ways that acknowledge women's realities, needs, and requests \\
\hline individualize Pap smear screening procedures to meet the needs and requests of women \\
\hline go step-by-step when performing Pap smear screening to inform women of the process \\
\hline identify women's strengths, successes, and goals with regards to Pap smear screening and life realities \\
\hline offer consistent and convenient screening times \\
\hline notify women of Pap screening results whether negative or positive \\
\hline $\begin{array}{l}\text { develop a Pap smear screening committee inclusive of Indigenous women, community members, healthcare providers, and other } \\
\text { stakeholders to inform education, decision-making, and screening practices }\end{array}$ \\
\hline
\end{tabular}

Table 3. Recommendations: Education for Pap smear screening with Indigenous Women

be educated about cultural safety in university education curricula, workshops, and by Indigenous community members.
be knowledgeable about professional cultural safety guidelines (e. g., Cultural Competence and Cultural Safety in Nursing Education - A
Framework for First Nations, Inuit and Métis Nursing, 2009 and First Nations, Inuit, Métis Health Core Competencies: A Curriculum
Framework for Continuing Medical Education, 2009).
have opportunities for clinical practice placements in Indigenous communities.
be educated about racism and stereotyping of Indigenous people in healthcare encounters and mainstream healthcare policies.
be educated about how to initiate conversations with Indigenous women about sensitive topics like sexual health and Pap smear screening.
be educated about providing trauma-informed care.

\section{Acknowledgements}

The first author would like to acknowledge the participation of the Healthcare providers in this study, Dr. Ruth Mattin-Misener (Dalhousie University), Dr. Annette Browne (University British Columbia), Dr. Marilyn MacDonald (Dalhousie University), Dr. Charlotte Loppie (University of Victoria) and my daughter, Madison Furlotte. The study received funding from the following: Atlantic Aboriginal Health Research Program (AAHRP); Psychosocial Oncology Research Training (PORT) Fellowship; Electa MacLennan Scholarship, Dalhousie University; AstraZeneca Rural Scholarship, Canadian Nurses Foundation; and Saint Francis Xavier University.

\section{REFERENCES}

[1] Aboriginal Nurses Association of Canada. (2009). Cultural competency \& cultural safety in nursing education: $A$ framework for First Nations, Inuit and Métis Nursing. Retrieved from http://www.anac.on.ca/competency.php.

[2] Ahmed, S., Shahid, R. K., \& Episkenew, J. A. (2015). Disparity in cancer prevention and screening in Aboriginal populations: Recommendations for action. Current Oncology, 22(6), 417-426.

[3] Anderson, J. M. (2002). Toward a post-colonial feminist methodology in nursing research: Exploring the convergence of post-colonial and black feminist scholarship. Nurse Researcher, 9(3), 7-27.

[4] Anderson, J. M. \& Reimer-Kirkham, S. (1999). Discourses on health: A critical perspective. In H. Coward \& P. Ratanakul (Eds.), A cross-cultural dialogue on health care ethics (pp. 47-67). Waterloo, ON, Canada: Wilfred Laurier University Press.

[5] Arnold, O. F., \& Bruce, A. (2005). Nursing practice with Aboriginal communities: Expanding worldviews. Nursing Science Quarterly, 18(3), 259-263.

[6] Atkinson, J. (2013). Trauma-informed services and trauma-specific care for Indigenous Australian children Retrieved from http://www.aihw.gov.au/closingthegap/

[7] Barnes, R., Josefowitz, N., \& Cole, E. (2006). Residential schools impact on Aboriginal students' academic and cognitive development. Canadian Journal of School Psychology, 21(1/2), 18-32.

[8] Barton, S. (2008). Discovering the literature on Aboriginal diabetes in Canada: A focus on holistic methodologies. Canadian Journal of Nursing Research, 40(4), 26-54.

[9] Battiste, M. (2000). Reclaiming indigenous voice and vision. Vancouver, BC, Canada: UBC Press.

[10] Black, A. T. (2009). Cervical cancer screening strategies 
for Aboriginal women. Pimatisiwin, 7(2), 157-179.

[11] Blix, B. H., Hamran, T., \& Normann, H. K. (2012). Indigenous life stories as narratives of health and resistance: A dialogical narrative analysis. Canadian Journal of Nursing Research, 44(2), 64-85.

[12] Brave Heart, M. Y. H., \& Daw, R. (2012). Welcome to Takini's historical trauma. Retrieved from http://historicaltrauma.com/.

[13] Browne, A. J. (2007). Clinical encounters between nurses and First Nations women in a Western Canadian hospital. Social Science and Medicine, 64(10), 2165-2176.

[14] Browne, A. J., Smye, V. L., Rodney, P., Tang, S. Y., Mussell, B., \& O'Neil, J. (2011). Access to primary care from the perspective of Aboriginal patients at an urban emergency department. Qualitative Health Research, 21(3), 333-348.

[15] Browne, A. J., Smye, V. L., \& Varcoe, C. (2005). The relevance of postcolonial theoretical perspectives to research in Aboriginal health. Canadian Journal of Nursing Research, 37(4), 16-37.

[16] Browne, A. J., \& Varcoe, C. (2006). Critical perspectives on culture and health in Aboriginal health. Contemporary Nurse (special edition): Advances in Indigenous Health Care, 22(2), 155-168.

[17] Browne, A. J., Varcoe, C. M., Wong, S. T., Smye, V. L., Lavoie, J., Littlejohn, D., \& Lennox, S. (2012).Closing the health equity gap: Evidence-based strategies for primary health care organizations. International Journal for Equity in Health, 11(59), 1-15.

[18] Canadian Cancer Society. (2012). What is cervical cancer? Retrieved

from http://www.cancer.ca/en/cancer-information/cancer-type/c ervical /overview/?region $=\mathrm{ns}$

[19] Canadian Institutes of Health Research. (2007). Guidelines for health research involving Aboriginal people. Retrieved from http://www.cihr-irsc.gc.ca/e/documents /ethics aboriginal guidelines_e.pdf.

[20] Cancer Care Ontario (2016). Cancer in First Nations in Ontario: Risk factors and screening. Retrieved from https://www.cancercareontario.ca/sites/ccocancercare/files/ assets/

CCOFNIMRiskFactorsReport2016.pdf?redirect=true

[21] Dion Stout, M. (2005). Healthy living and Aboriginal women: The tension between hard evidence and soft logic. Centres of Excellence for Women's Health Research Bulletin. Retrieved from http://www.findarticles.com/p/articles/mi_qa4118

[22] Dion Stout, M. (2012). Ascribed health and wellness, Atikowisi miýw- ay awin, to achieved health and wellness, Kaskitamasowin miýw-a ay awin: Shifting the paradigm. Canadian Journal of Nursing Research, 44(2), 11-14.

[23] First Nations and Inuit Health. (2012). Family health. Retrieved http://www.hcsc.gc/fniah-slina/famil/index-eng.php

[24] Fiske, J., \& Browne, A. (2006). Aboriginal citizen, discredited medical subject: Paradoxical constructions of Aboriignal women's subjectivity in Canadian health care policies. Policy Science, 39(1), 91-111.

[25] Garman, A. \& Doull, K. (2009). Embracing the healing journey. Journal of Aboriginal Health, 5(1), 2-3.

[26] Iwama, M., Marshall, M., Marshall, A., \& Bartlett, C. (2009). Two-eyed seeing and the language of healing in community-based research. Canadian Journal of Native Education, 32(2), 3-23.

[27] Koea, J. B. (2008). Indigenous trauma: A New Zealand perspective. Injury: International Journal of the Care of the Injured, 39(5), S11-S18.

[28] Knockwood, I. (2001). Out of the depths: The experiences of Mi'Kmaw children at the Indian residential school at Shubenacadie, Nova Scotia. Black Point, NS, Canada: Roseway Publishing.

[29] Letendre, A. (2008). Aboriginal female sexual health in a context of cervical cancer and cervical cancer cytology screening with reference to the Cree and Cree-Metis of Northern Alberta. (Doctoral Dissertation). University of Alberta, Edmonton, Canada.

[30] Lewis, R., \& Mills, S. (2003). Feminist postcolonial theory: $A$ reader. New York, NY: Routledge.

[31] Loppie, C. (2007). Learning from the grandmothers: Incorporating indigenous principles into qualitative research. Qualitative Health Research, 17(2), 276-284.

[32] Loppie-Reading, C., \& Wien, F. (2009). Health inequalities and social determinants of Aboriginal peoples health. Prince George, BC, Canada: National Collaborating Centre for Aboriginal Health.

[33] Maar, M., Burchell, A., Little, J., Ogilivie, G. Severini, A, Yang, J. M. \& Zehbe, I. (2013). A qualitative study of provider perspectives of structural barriers to cervical cancer screening among First Nations women. Women's Health Issues, 23(5), e319-e325.

[34] MacDonald, C. (2008). Using components of the medicine wheel for the development of a conceptual framework for understanding Aboriginal women in the context of Pap smear screening. Pimatisiwin Journal: A Journal of Aboriginal and Indigenous Community Health, 6(3), 95108.

[35] MacDonald, C. (2012). Understanding participatory action research: A qualitative research methodology option. The Canadian Journal of Action Research, 13(2), 35-40.

[36] MacDonald, C. (2015). Martin-Misener, R. Steenbeek, A., \& Browne, A. (2015). Honoring Stories: Mi'kmaq women's Experiences with Pap Smear Screening in Eastern Canada. Canadian Journal of Nursing Research, 47(1), 72-96.

[37] Mancini, R. (2007). Telling their stories. Education Forum, $33(2), 15-18$.

[38] Martin, D. (2012). Two-Eyed Seeing: A framework for understanding Indigenous and non-Indigenous approaches to Indigenous health research. Canadian Journal of Nursing Research, 44(2), 20-42.

[39] McGuire, P. D. (2010). Exploring resilience and indigenous ways of knowing. Pimatisiwin, 8(2), 117-131. 
[40] Morgan, L., \& Wabie, J. L. (2012). Aboriginal Women's access and acceptance of reproductive health care. Pimatisiwin: A Journal of Aboriginal and Indigenous Community Health, 10(3), 313-325.

[41] National Aboriginal Health Organization. (2010). Cervical cancer in First Nations women: Information for health care providers. Ottawa, ON, Canada.

[42] O’Brien, B. A., Mill, J., \& Wilson, T. (2009). Cervical cancer screening in Canadian First Nation Cree women. Journal of Transcultural Nursing, 20(1), 83-92.

[43] O'Connor, H., \& Gibson, N. (2003). A step -by-step guide to qualitative data analysis. Pimatisiwin: A Journal of Aboriginal and Indigenous Community Health, 1(1), 6490 .

[44] Palacios, J. F., \& Portillo, C. J. (2009). Understanding Native women's health: Historical legacies. Journal of Transcultural Nursing, 20(1), 15-27.

[45] QSR International. (2007). N-Vivo. Retrieved from http://www. qsrinternational.com/products_nvivo.aspx

[46] Ralph, N., Hamaguchi, K., \& Cox, M. (2006). Transgenerational trauma, suicide and healing from sexual abuse in Kimberley, Australia. Pimatisiwin Journal: A Journal of Aboriginal and Indigenous Community Health, 4(2), 117-136.

[47] Reimer-Kirkham, S., \& Anderson, J. M. (2002). Postcolonial nursing scholarship: From epistemology to method. Advances in Nursing Science, 25(1), 1-17.

[48] Sandelowski, M. (1995). Qualitative analysis: What is it and how to begin. Research in Nursing \& Health, 18(4), 371-375.

[49] Schnarch. B. (2005). Ownership, control, access, and possession (OCAP) or self-determination applied research: A critical analysis of contemporary First Nation research and some options for First Nation communities. Retrieved from

http://www.research.utoronto.ca/ethics/pdf/human/nonspec ific/ OCAP\%20principles.pdf

[50] Shadhid, S., Finn, L., \& Thompson, S. C. (2009). Barriers to participation of Aboriginal people in cancer care. $M J A$, 190(10), 574-579.

[51] Sherwood, J., \& Edwards, T. (2006). Decolonisation: A critical step for improving Aboriginal health. Contemporary Nurse, 22, 178-190.

[52] Smith, D., Edwards, N., Peterson, W., Jaglarz, M., Laplante, D., \& Estable, A. (2010). Rethinking nursing best practices with Aboriginal communities: Informing dialogue and action. Nursing Leadership, 22(4), 24-39.

[53] Streubert, H. J., \& Carpenter, D. R (1995). Qualitative research in nursing: Advancing the humanistic imperative. Philadelphia, PA: Lippincott.
[54] Trees, J. (2008). Cultural and historical factors in Aboriginal women's sexual health. Issus, 83, 17-20.

[55] Vollman, A. R., Anderson, E. T., \& McFarlane, J. (2004). Canadian community as partner. Philadelphia, PA: Lippincott Williams \& Wilkins.

[56] Vukic, A., Gregory, D., \& Martin-Misener, R. (2012). Indigenous health research: Theoretical and methodological perspectives. Canadian Journal of Nursing Research, 44(2), 146-161.

[57] Watson, M, Bernard, V., Thomas, C., Brayboy, A., Paisano, R. \& Becker, T. (2014). Cervical Cancer Incidence and Mortality Among American Indian and Alaska Native Women, 1999-2009. American Journal of Public Health, 104(S3), S415-S422.

[58] Warry, W. (2007). Ending denial: Understanding Aboriginal issues. Peterborough, ON, Canada: Broadview Press.

[59] Weber-Pillwax, C. (2004). Indigenous research and indigenous research methods: Cultural influences or cultural determinates of research methods. Pimatisiwin, 26(1), 77-90.

[60] Weedon, C. (2002). Key issues in postcolonial feminism: A Western perspective. Gender Forum. Retrieved from http://www.genderforum.org/issues/genderealisations / key-issues-in-postcolonial-feminism-a-western-perspective

[61] Whitty-Rogers, J. Cameron, B. \& Caine, V. (2020). An Indigenous and Western paradigm to understand gestational diabetes mellitus: Reflections and insights. Action Research, 0(0), 1-22.

[62] Wilk, P., Maltby, A. \& Cooke, M. (2017). Residential schools and the effects on Indigenous health and well-being in Canada-A scoping review. Public Health Review 38(8), $1-23$

[63] Wilson, D. (2008). The significance of a culturally appropriate health service for indigenous Maori women. Contemporary Nurse, 28, 173-188.

[64] Young, T. K., Kliewer, E., Blanchard, J., \& Mayer, T. (2000). Monitoring disease burden and preventive behavior with data linkage: Cervical cancer among Aboriginal people in Manitoba, Canada. American Journal of Public Health, 90(9), 1466-1468.

[65] Zehbe, I., Maar, M., Nahwegahbow, A. J., Berst, K. S. M., \& Pintar, J. (2012). Ethical space for a sensitive research topic: Engaging First Nations women in the development of culturally safe human papillomavirus screening. Journal of Aboriginal Health, 8(1), 41-50.

[66] Zehbe, I., Wakewich, P., Wood, Briabbe, Sameshima, P, Banning, Y. \& Little, J. (2016). Engaging Canadian First Nations women in cervical screening through education. International Journal Health Promotion Education, 54(5), 255-264. 